\title{
The Power of Movement: Evidence for Context-Independent Movement Imitation
}

\author{
Oliver Genschow \\ University of Mannheim
}

\author{
Arnd Florack \\ University of Vienna
}

\author{
Michaela Wänke \\ University of Mannheim
}

\begin{abstract}
Recent studies have shown that individuals often imitate the behavior of others. In these studies, the observed and imitated behaviors were always identical. The present research goes one step further and disentangles the imitation of movements from their behavioral contexts. On the basis of theories that the perception of behavior refers to the same mental representations as the execution, we found that imitation is not confined to the same class of behaviors but rather to the same class of movements that may be involved in different behaviors. Four studies demonstrated that watching an athlete lifting a barbell leads to an increase in participants' drink intake when drinking involved a similar movement (lifting a cup) but not when drinking did not involve a lifting movement (drinking through a tube). The effects were stronger for individuals high in perspective taking (Study 1) and for situations in which the perspective was manipulated to be similar to the observed actor's (Study 2). These findings demonstrate the power of movements in imitation processes, suggesting that shared goal representation is not necessary for imitating others' movements.
\end{abstract}

Keywords: imitation, motor mimicry, stimulus-response compatibility, goal independent imitation

People tend to adjust their drink and food intake to others' consumption. For instance, Herman, Koenig-Nobert, Peterson, and Polivy (2005) offered pairs of participants pizza slices and found that the number of slices eaten by one participant in a pair correlated highly with the number of pizza slices eaten by the second participant. Other studies (Conger, Conger, Costanzo, Wright, \& Matter, 1980; Goldman, Herman, \& Polivy, 1991; Johnston, 2002; Nisbett \& Storms, 1974) have applied similar designs, but instructed one participant or a confederate in each pair to consume either a small (e.g., one cracker) or a large amount of food (e.g., 20 crackers). Again, a consistent finding was that the amount of food participants consumed depended strongly on the consumption of the confederate.

A prominent explanation suggests that motivated orientations toward social norms (for a review, see Herman, Roth, \& Polivy,

This article was published Online First September 3, 2012

Oliver Genschow, Department of Consumer and Economic Psychology, University of Mannheim, Mannheim, Germany; Arnd Florack, Department of Psychology, University of Vienna, Vienna, Austria; Michaela Wänke, Department of Consumer and Economic Psychology, University of Mannheim.

We would like to thank Klaus Fiedler for his helpful comments on an earlier version of this article; Bruno Wickli for his support regarding the videos; and Judith Tonner, Julian Däster, Jana Keil, and Carina Enzenhofer for their help in collecting the data.

Correspondence concerning this article should be addressed to Oliver Genschow, Department of Consumer and Economic Psychology, University of Mannheim, Parkring 47, 68159 Mannheim, Germany. E-mail: ogenscho@mail.uni-mannheim.de
2003) account for this phenomenon. Although the motivation to strive for a positive evaluation by others and to avoid a negative evaluation is reasonable, alternative or additional accounts may also play a role. A particularly intriguing question is whether more basic mechanisms of movement imitation facilitate the modeling of consumption behavior. Research on imitation (e.g., Brass \& Heyes, 2005; Heyes, 2001; for a review, see Meltzoff \& Prinz, 2002) has repeatedly demonstrated that perceiving an action facilitates the execution of a similar action. Thus, it is plausible to assume that also the imitation of specific movements (i.e., lifting one's hand to one's mouth) contributes to the convergence in eating and drinking behavior. However, as long as the observed movement (e.g., arm lifting) occurs within the imitated behavioral context (e.g., drinking), it is difficult to test the impact of mere movement imitation. To test the power of movement imitation, one needs to separate the crucial movement from its context. Presumably, movements that are similar to those needed for the execution of consumption behavior should make consumption behavior more likely, even when they are executed in another context with a different goal. For instance, the movement involved in lifting a cup to one's lips to drink out of it is similar to the movement involved in lifting a barbell in weight training. Following this line of reasoning, the purpose of the present research was to examine whether observing a specific movement (e.g., lifting a barbell) would also affect another behavior that requires the same movement but with a different goal (i.e., drinking).

\section{Theoretical Background}

Previous research has not yet examined the question of whether the observation of movements affects similar movements in an- 
other behavioral context performed with a different goal. However, two lines of related research are particularly relevant to this question-yet suggest contrary answers. The first line of research implies that individuals are more likely to adopt the goal of a model than the exact manner in which the goal is realized. For a child, for example, it is more important to make a rattle clatter than to rattle it with the same hand movements as the parent. Indeed, the idea that goal direction is the central feature of imitation has been prominent in research with children (Bekkering, Wohlschläger, \& Gattis, 2000; Carpenter, Akhtar, \& Tomasello, 1998; Gergely, Bekkering, \& Kiraly, 2002; Huang \& Charman, 2005; Meltzoff, 1995) as well as with nonhuman primates (Byrne, 2003; Byrne \& Byrne, 1991). According to this research, the adoption of a goal representation activates a motor program that is most commonly and easiest to use independently of whether this movement matches the movement performed by a model (Bekkering et al., 2000; Gattis, Bekkering, \& Wohlschläger, 2002; Gleissner, Bekkering, \& Meltzoff, 2000). This notion is consistent with past (e.g., Powers, 1973) but also with more recent models (e.g., Bargh \& Ferguson, 2000), suggesting that the key driver of human behavior is its underlying goal.

There is no doubt that the adoption of goals is one of the most central factors of (imitative) behavior. But it is also important to take into consideration the motor actions that are needed to reach a goal. For example, if a basketball player has the goal of shooting the ball into the basket, the goal itself will not result in making the basket. To make the basket, it is important to control the respective motor actions needed for shooting the ball properly-otherwise it is impossible to get the ball through the hoop. Even when mentally simulating an action, recent theories in embodied cognition such as the perceptual symbol systems (Barsalou, 1999; Niedenthal, Barsalou, Winkielman, Krauth-Gruber, \& Ric, 2005) would suggest that not only the goal of an action but also the physical control over the action is of central importance.

Here a second line of research comes into play. Research on imitation and mimicry ${ }^{1}$ provides hints that a crucial factor for imitating a specific action may lie in processes directly concerning the execution of a goal. It has been shown that movements can be primed and are more likely to be executed when the respective action is observed without assuming that a shared goal representation is necessary. For instance, simple mannerisms like foot waggling, nose rubbing, or postures are often directly imitated (cf. Chartrand \& Bargh, 1999; for a review, see Chartrand, Maddux, \& Lakin, 2005). More specifically, it has been demonstrated that observing compatible movements facilitates the execution of these movements and observing incompatible movements impedes the execution of such movements (Brass, Bekkering, \& Prinz, 2001; Brass, Bekkering, Wohlschläger, \& Prinz, 2000). For example, in one experiment, participants observed a video of an actor either lifting his or her index finger up or tipping it down. Participants were instructed to respond either with finger lifting or tipping as soon as the video appeared on the screen. The results indicated that the observation of movements compatible with the instructed movements resulted in faster movement onsets than the observation of incompatible movements (Brass et al., 2001). Going one step further, Leighton and Heyes (2010) elegantly showed that not only the goal of an action but also the concrete movement is of importance. In their experiments, the task of participants was either to open or to close their hand or their mouth in response to letters presented on a screen. Importantly, the same screen showed pictures of mouths or hands that varied with regard to whether they were opened or closed. Interestingly, the authors found that participants were influenced not only by the presented goal (opening or closing) across effectors (hand or mouth), but mostly by the specific effector that was needed for the execution.

An interesting explanation of such forms of imitation was already given by William James (1890) more than 100 years ago. With regard to what he referred to as ideomotor action, he maintained that "every representation of a movement awakens in some degree the actual movement which is its object" (James, 1890, p. 1134). Based on this idea, ideomotor theory (Brass et al., 2001, 2000; Greenwald, 1970; Jeannerod, 1999; Prinz, 1990, 1997) postulates that actions are represented in terms of the sensory feedback they produce. Moreover, the observation of a certain action primes the execution of the same action because both actions involve the same sensory mechanisms. Likewise, research in social psychology explains phenomena such as mimicry with reference to a perception-behavior link (Chartrand \& Bargh, 1999; Chartrand et al., 2005; Dijksterhuis \& Bargh, 2001; Dijksterhuis, Smith, van Baaren, \& Wigboldus, 2005). This link basically assumes that perceiving an action evokes the same representation as the execution of this action and thereby makes the execution of the perceived action more likely. The idea of such shared representational systems has also received empirical support from neurophysiological as well as brain image research. For example, Berger and Hadley (1975) already found more than 3 decades ago that observing motor behavior leads to corresponding muscle activations in the observer. The researchers placed a set of electrodes on participants' arms to measure muscle activity when participants watched an arm-wrestling match. They found that participants who watched the arm-wrestling match showed stronger electromyographic activity in their own forearms and wrists than participants who observed an unrelated behavior. Fadiga, Fogassi, Pavesi, and Rizzolatti (1995) reported similar findings. They recorded motorevoked potentials from various hand and arm muscles while participants watched an experimenter grasping an object. The observed motor-evoked potential pattern was similar to that of participants executing the movement. Further support has been provided by brain-imaging research, suggesting that the perception of behavior leads to activations in the brain similar to those activated by the execution of the behavior (for an overview, see Rizzolatti \& Sinigaglia, 2010). For example, areas such as the cerebellum (Grossman et al., 2000) and the posterior parietal cortex (Ruby \& Decety, 2001) show similar activation when performing or when observing a specific action (for a more critical view see, Lingnau, Gesierich, \& Caramazza, 2009).

The notion that observing a specific movement will activate the respective motor system and facilitate the execution of the observed movement suggests that goal representation may not be necessary for imitating a movement. Indeed, ideomotor theory

\footnotetext{
${ }^{1}$ In contrast to imitation, mimicry is detected in naturalistic social situations and is prominent in social psychology research. Imitation, on the other hand, can be regarded as the laboratory model of mimicry and is mostly studied in experimental psychology. Although there is little integrative research on imitation and mimicry, it has been widely suggested that the two phenomena depend on the same psychological and neural processes (for a review, see Heyes, 2011).
} 
(Brass et al., 2001, 2000; Greenwald, 1970; Jeannerod, 1999; Prinz, 1990, 1997) and research on the perception-behavior link (Chartrand \& Bargh, 1999; Chartrand et al., 2005; Dijksterhuis \& Bargh, 2001; Dijksterhuis et al., 2005) suggest that the mere observation of a specific movement facilitates the execution of the same movement without assuming that observers have to adopt the goal of the actor. However, a crucial test of whether imitation of specific movements without adopting a similar goal is possible would require disentangling the observed movement from the observed behavioral goal. On the basis of findings that the observation of a movement activates motor programs similar to the performance of the movement, one would predict that imitation is not limited to the observed behavior (e.g., drinking), but may facilitate any behavior that involves the observed movement (e.g., arm lifting). This should even then be the case if the movement is conducted in a completely different behavioral context (e.g., weight training).

Our first aim is to provide a strong test for theories proposing an overlap between movement observation and movement execution (i.e., ideomotor theory and the perception-behavior link). If our assumption is correct, the notion that individuals imitate movements across contexts and goals would highlight the importance of the role of motor actions in imitation processes. A second aim of our research addresses a different and more methodological issue. Most previous research on ideomotor theory has involved movements that were isolated from goal-related, world behavioral contexts and detached from the behavior's social context. For instance, participants lifted their finger up or tipped it down when observing a finger movement that was compatible or incompatible with their own finger movement (e.g., Brass et al., 2001). Our goal was to examine the hypothesis within a social and a meaningful behavioral context—namely, drink consumption. More precisely, we aimed to test whether individuals' drink intake would increase when they observed a movement that was similar to a drinking movement (arm lifting) but was executed in another behavioral context (weight training) and with a different goal (muscle exercise).

\section{Overview of the Research}

To examine our hypotheses, we conducted four studies. In all studies, participants were asked to taste and evaluate a sports drink while they watched a video. The video showed an athlete exercising with a barbell. The movement was either compatible (arm lifting) or incompatible (arm pushing) with the movement needed to bring a cup from a table to the mouth (drinking movement). We expected that observing the compatible movement would facilitate lifting a cup compared to observing the incompatible movement. Of course, lifting the cup is only one element in an action sequence. However, we assumed that once the observed movement initiated the motor program, it would be carried out. In sum, we expected that in a situation that affords drinking (because glasses filled with a beverage are present), observing arm lifting would enhance drink intake compared to observing a movement that was incompatible with a drinking movement.

In addition, in Studies 1 and 2, we were interested in whether perspective taking would facilitate the effect of the observation of a movement on the execution of the same movement in a different context. Previous research has shown that perspective taking mod- erates perception-behavior effects (e.g., Chartrand \& Bargh, 1999), indicating that individuals who are good at taking the perspective of others are more likely to imitate others than those who are not good at taking the perspective of others. Therefore, we expected stronger effects of the observation of the movements on drink intake when participants adopted the perspective of the observed model. In Study 1, we tested whether the extent to which participants could take the perspective of the model would moderate the predicted effects. In Study 2, we manipulated perspective taking experimentally. In one condition, participants watched the video from a first-person perspective to adopt the perspective of the model, but in the other condition, participants watched the video from a third-person perspective.

Whereas Studies 1 and 2 attempted to find the first evidence that the imitation of movements across contexts and independent of shared goals is possible, Studies 3 and 4 were designed to shed light on the underlying processes. Study 3 assessed whether increased drink intake was really due to more frequent lifting of drinking cups rather than merely larger sips. Finally, Study 4 tested the specificity of the imitated action and addressed a possible alternative account, namely, the priming of approach versus avoidance concepts. If our assumption is correct, watching someone lifting barbells should increase drink intake only when the drinking behavior is realized by a similar motor action (lifting cups) but not when drinking involves a behavior unrelated to the observed movement (drinking from a tube). By contrast, if watching arm flexion primes approach behavior and arm pushing primes avoidance behavior, the effect should not be limited to the specific motor action by which drinking is realized.

\section{Study 1}

In Study 1, we presented participants with a video of an athlete in a gym either lifting or pushing a barbell and, at the same time, asked them to test a beverage. The lifting movement is compatible to a drinking movement because it involves the same muscles as the movement needed for bringing a cup from a table to the mouth. By contrast, the pushing movement is incompatible with the typical drinking movement. As outlined above, we expected that cup lifting would be facilitated and that participants would drink more of the beverage when they watched an athlete lifting a barbell compared to watching an athlete pushing a barbell. However, because perspective taking is a crucial precondition in imitation, we additionally measured the extent to which participants adopted the perspective of the athlete and expected that the effect would be moderated by perspective-taking abilities. We therefore formulated the following hypothesis:

Hypothesis 1: Watching an athlete lift a barbell will increase drinking the more participants take the athlete's perspective.

\section{Method}

Participants and design. Fifty-eight female students were randomly assigned to one of the two video conditions: compatible drinking movement versus incompatible drinking movement. They received partial course credit in exchange for their participation. Data from seven participants were excluded from the analysis because the participants had consumed more than $2,000 \mathrm{ml}$ of 
standard alcoholic beverages within $24 \mathrm{hr}$ before the experiment and thus had a potentially increased thirst level (e.g., Montastruc, 1986; Nicholson \& Taylor, 1938) or they had emptied the offered beverage after observing the video (and so their drink intake while watching the movement could not be measured; see below). These selection criteria were used in all studies reported in this article. The age of the final sample ranged from 19 to 25 years $(M=$ 21.54, $S D=1.43$ ).

Procedure. After being greeted and seated at a table with a desktop computer, participants signed a statement of agreement. As a cover story, the experimenter told the participants that a new sports drink that was designed for consumption during workouts in fitness studios and health clubs would be introduced. The participants' task would be to taste and rate the drink. The experimenter also explained that in order to simulate the context of working out, a video of an athlete exercising with a barbell would be presented while participants tasted the drink. To make sure that every participant had an equal level of thirst, the experimenter asked participants to drink from a $300 \mathrm{ml}$ plastic cup of water until they were not thirsty anymore. Then the participants started the experiment on the computer, indicated the amount of alcohol consumed during the last $24 \mathrm{hr}$, and indicated basic demographic characteristics. After answering these questions, participants watched two times a video of an athlete exercising with a barbell. For the first video ( $24 \mathrm{~s}$ ), the participants were instructed just to watch the video. For the second video, the experimenter offered participants a transparent plastic cup with $300 \mathrm{ml}$ of a green-tea-flavored mineral water, which was presented as the new sports drink. No brand name was given. The experimenter told the participants that they could drink as much as they wanted while watching the same video of an athlete exercising with the barbell. The video was taken from a third-person perspective and lasted 40 s. Participants in the compatible movement condition watched the male athlete filmed from the front, standing upright, and lifting a barbell from his waist to his chest. Participants in the incompatible movement condition watched the same athlete filmed from above lying on his back, pushing the barbell up from his chest. In both videos, the athlete moved the barbell eight times with the same rhythm. After watching the video, participants indicated on three 9-point scales $(1=$ not at all, $9=$ very much $)$ their degree of perspective taking while watching the video ("I could put myself in the athlete's place," "I could empathize with the athlete's effort," "I could almost feel the effort in my own muscles"). ${ }^{2}$ Afterward, participants rated the drink on three items ("I like this sports drink," "The sports drink appeals to me," "I would recommend this sports drink to a friend") on 9-point scales $(1=$ not at all, $9=$ very much $)$ as part of the cover story. Finally, participants were probed for suspicion, debriefed, and dismissed. To measure the consumed portion of the drink, we weighed each cup before and after consumption.

\section{Results}

Drink intake. None of the participants correctly guessed our hypothesis. To test our assumption, we averaged the three questions on perspective taking into a single score of perspective taking (Cronbach's $\alpha=.91$ ). We expected that perspective taking would moderate the impact of the watched movement (compatible arm movement vs. incompatible arm movement) on drink intake. To test our hypothesis, we ran a multiple regression analysis $\left(R^{2}=\right.$ .12). First, all continuous variables were $z$ standardized (Aiken $\&$ West, 1996). The amount of the beverage consumed served as the dependent measure. As predictors, we entered the dummy-coded movement $(1=$ compatible arm movement, $0=$ incompatible arm movement), perspective taking, and the interaction between these variables. As expected, the interaction between movement and perspective taking was statistically significant, $\beta=.35, t(37)=$ $2.20, p=.03$, indicating that the better participants were at taking the perspective of the athlete, the more they drank from the offered drink in the compatible movement condition compared to the incompatible movement condition. The main effects of movement $\left(M_{\text {compatible }}=62.43 \mathrm{~g}, S D=62.53 ; M_{\text {incompatible }}=66.25 \mathrm{~g}, S D=\right.$ $44.62)^{3}$ and of perspective-taking abilities were not significant $(\beta \mathrm{s}<.09, t \mathrm{~s}<0.56, p \mathrm{~s}>.58)$. Simple slope tests (Aiken \& West, 1996) for the two experimental conditions further supported the hypothesis that the effect of movement compatibility on drink consumption increased with an increase in perspective taking. When participants watched the athlete executing the compatible arm movement, participants drank more of the beverage the better they could take the perspective of the athlete, $\beta=.46, t(37)=$ $2.02, p=.05$. By contrast, when participants watched the athlete executing the incompatible arm movement, perspective taking was not correlated with drink intake, $\beta=.22, t(37)=1.06, p=.29$.

Drink evaluation. In a further analysis, we tested whether the movements that were viewed and perspective taking had effects on drink evaluation. We first averaged the three questions on drink evaluation into a single scale (Cronbach's $\alpha=.96$ ) and then computed a multiple regression analysis $\left(R^{2}=.02\right)$. As predictors, we entered the dummy-coded movement $(1=$ compatible arm movement, $0=$ incompatible arm movement), perspective-taking abilities, and the interaction between these variables. The averaged drink evaluation served as the dependent measure. Neither the main effects nor the interaction reached conventional levels of significance $(\beta \mathrm{s}<.20, t \mathrm{~s}<0.85, \mathrm{ps}>.40)$.

\section{Discussion}

Study 1 demonstrated that the better the participants were at adopting the perspective of the actor, the more they drank from the beverage while they watched the actor execute a movement compatible with a drinking movement compared to a movement incompatible with a drinking movement. Study 1, thus, supports the prediction that an action is facilitated when a similar movement is observed. Importantly, to the best of our knowledge, this is the first study showing that the observation of a movement in a different context and with a different goal can be imitated. Whereas the actor performed arm lifting in the context of weight training with the goal of exercising his muscles, the participants lifted cups to their mouths with the goal of tasting a drink. These results support previous theories proposing that the observation of a motor movement will lead to an activation of the motor system in the observer

\footnotetext{
${ }^{2}$ German translations of the items are "Ich konnte mich in die Situation des Sportlers hineinversetzen," "Ich konnte die Anstrengung des Sportlers nachempfinden," "Ich konnte die Anstrengung in den beanspruchten Muskeln förmlich spüren."

${ }^{3}$ Regression analyses were conducted with $z$-transformed values. How ever, untransformed values were reported to facilitate the interpretation.
} 
that is similar to executing the movement. Moreover, in line with recent research on imitation (e.g., Brass \& Heyes, 2005; Leighton \& Heyes, 2010), our results support the idea that a shared goal representation is not necessary for imitating a specific movement.

The finding that perspective taking moderates the effects of observation on movement execution is in line with previous findings that have shown that perspective taking is a precondition for imitation to occur (Chartrand \& Bargh, 1999). However, in Study 1 , perspective taking was measured but not manipulated. Nevertheless, we assume that, independent of existing interindividual differences, situations that foster perspective taking are also more likely to elicit the effect of the observation on the execution of a movement. Hence, in Study 2, we aimed to replicate the results of Study 1 by manipulating the perspective in the videos.

\section{Study 2}

The objective of Study 2 was to replicate the results of Study 1 when the perspective was manipulated. Studies in neuroscience and social psychology suggest not only that perspective taking is an individual difference variable, but that the experiences of others are more likely to affect an observer when the context induces a first-person perspective compared to the typical third-person perspective (Jackson, Meltzoff, \& Decety, 2006; Lamm, Batson, \& Decety, 2007; Storms, 1973). Accordingly, in Study 2, we presented videos to participants from either a first-person or a thirdperson perspective. We assumed that the presentation of the video from the first-person perspective would help participants to better put themselves into the actor's situation compared to the thirdperson perspective. Consequently, in this condition, watching the actor executing the lifting movements (compatible) compared to the pushing movements (incompatible) was expected to promote drinking. In sum, we formulated the following hypothesis:

Hypothesis 2: Compared to watching a third-person perspective video, watching a first-person perspective video will lead to an increase in compatibility effects on drink consumption (participants will drink more when they observe an athlete performing arm lifting than when they observe the same athlete performing arm pushing).

\section{Method}

Participants. Participants were 101 students (31 men and 70 women). They were recruited in exchange for partial course credit and tested in groups of up to six persons. The ages of participants ranged from 18 to 32 years with a mean age of $21.85(S D=2.67)$.

Procedure. The procedure of Study 2 was similar to that of Study 1. However, in contrast to Study 1 , half the participants watched the same videos of the athlete from the third-person perspective used in Study 1, and the other half watched the videos from a first-person perspective such that only the arms of the athlete and the barbell were visible (see Figure 1). The variations of the movements were the same as in Study 1. Whereas participants in the compatible arm movement condition saw the athlete's arms lifting a barbell, participants in the incompatible arm movement condition saw the athlete's arms pushing the barbell up. In all videos, the athlete's rhythm of pushing or lifting the barbell and the duration (40 s) were identical. While watching the video,

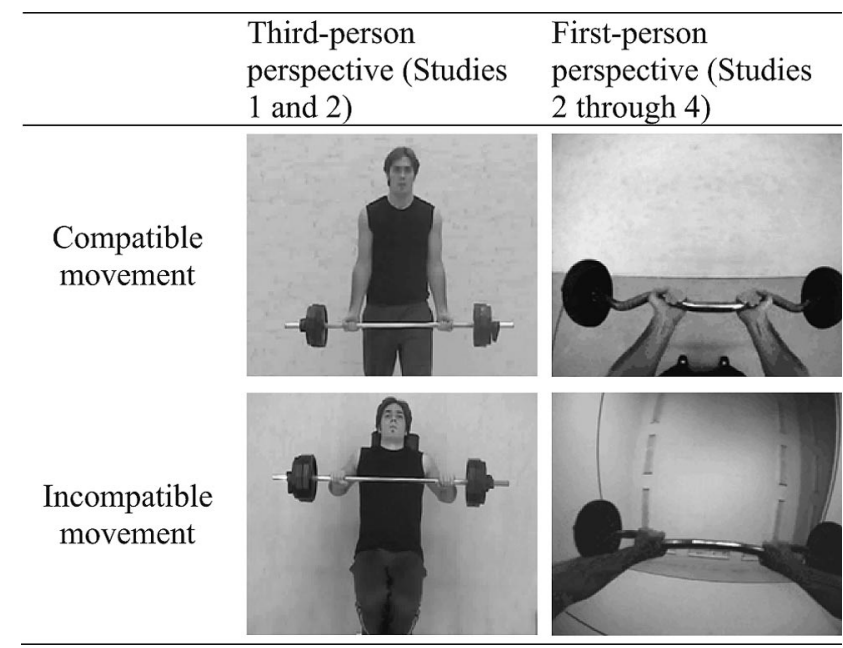

Figure 1. Screenshots of the third-person perspective videos used in Studies 1 and 2 and of the first-person perspective videos used in Studies $2-4$.

participants were instructed to taste a green-tea-based pricklypear-flavored drink. Afterward, participants rated the drink on the same items used in Study 1, were probed for suspicion, debriefed, and dismissed.

\section{Results}

Drink intake. None of the participants correctly guessed the purpose of the experiment. We had expected that the effect of higher drink intake while observing a compatible rather than an incompatible arm movement would be more pronounced when participants observed the action from a first-person perspective compared to a third-person perspective. Consistent with that hypothesis, a 2 (perspective: third person vs. first person) $\times 2$ (movement: compatible vs. incompatible) analysis of variance (ANOVA) with drink intake as the dependent variable showed a significant interaction between perspective and movement, $F(1$, $96)=5.97, p=.02, \eta_{\mathrm{p}}^{2}=.059$. In the first-person perspective condition, participants consumed more of the drink when they watched the compatible arm movement $(M=114.28 \mathrm{~g}, S D=$ 71.16) than when they watched the incompatible arm movement $(M=76.52 \mathrm{~g}, S D=43.67), t(97)=2.26, p=.03, d=0.65$. From the third-person perspective, the observed arm movements did not influence drink intake $\left(M_{\text {compatible }}=84.40 \mathrm{~g}, S D=48.37\right.$; $\left.M_{\text {incompatible }}=103.65 \mathrm{~g}, S D=67.20\right), t(97)=-1.17, p=.25$. Also, the main effects of perspective, $F(1,96)=0.01, p=.94$, and observed movement, $F(1,96)=0.67, p=.42$, were not significant.

Drink evaluation. In a further analysis, we tested whether the perspective of the videos and the observed movement had an effect on the evaluation of the drink. First, the three drink evaluation items were summed into a single scale (Cronbach's $\alpha=.97)$. We then computed a 2 (perspective: third person vs. first person) $\times 2$ (movement: compatible vs. incompatible) ANOVA with drink evaluation as the dependent variable. Neither the interaction between perspective and observed movement nor any of the main effects were significant, $F \mathrm{~s}(1,96)<0.54, p \mathrm{~s}>.46$. 


\section{Discussion}

In line with Study 1, the results of Study 2 suggest that the observation of a movement unrelated to consumption boosts consumption when the observed movement is similar to the consumption movement and individuals adopt the perspective of the actor. When participants observed an athlete exercising with a barbell from the first-person perspective, they consumed more of a drink when the athlete executed a movement compatible with a typical drinking movement than when the athlete executed a movement incompatible with the drinking movement. By contrast, when participants observed the athlete from a third-person perspective, no differences between the observations of the two movements occurred. These results replicate the results of Study 1, which showed that the compatible movement facilitated drinking the better participants were at taking the perspective of the athlete.

It is important to note that Studies 1 and 2 congruently support the assumption that perspective taking is a precondition for movement compatibility effects on drinking consumption. The main difference between Studies 1 and 2 was the method used to test the moderating effect of perspective taking. Whereas we measured the degree of participants' perspective taking in Study 1, we manipulated perspective taking by presenting the videos from a firstperson or third-person perspective in Study 2. The results were identical in the two studies. Neither study showed a main effect of movement compatibility, but both studies found an interaction between movement compatibility and the adopted perspective. Drink intake increased when observing arm flexion compared to observing arm extension primarily when observers took the perspective of the observed actor-either spontaneously (Study 1) or because of the manipulated perspective (Study 2). Jointly, the results of the two studies are in line with previous studies in showing that imitation is more likely to occur when individuals take the perspective of another person (Chartrand \& Bargh, 1999; Jackson et al., 2006; Lamm et al., 2007; Lamm, Porges, Cacioppo, \& Decety, 2008).

We argue that an imitation account underlies our previous findings. Concretely, we assume that observing a compatible movement (arm lifting) facilitated the execution of the same movement (arm lifting for drinking), which resulted in an increased drink intake. At this point, however, there are two critical issues regarding this explanation. First, we have to acknowledge that we only have indirect evidence for imitated arm lifting. We did not measure how often participants raised their hands to their mouths but interpreted increased drink intake as a proxy for imitated movements. We had assumed that imitation would induce more frequent arm lifting, which then would also entail increased drink intake. In principle, however, one might contend that simply larger sips could account for increased drink intake. Such an alternative account might be based on the notion that the observed movements primed broad schemata of approach and avoidance. Watching a model lifting something toward the body could prime an approach schema, and watching a model pushing something away might conversely prime an avoidance schema. To gain more direct evidence for our assumption of facilitated arm lifting movements through the observation of a compatible movement, we conducted Study 3 in which we assessed arm lifting directly. However, evidence for more frequent arm lifting itself would not rule out the possibility that observing arm lifting may induce approach behav- ior and therefore instigate more frequent arm lifting and drinking. To address this second objection in a more straightforward fashion, we conducted Study 4.

\section{Study 3}

The results of Studies 1 and 2 do not unambiguously show whether the increased drink intake was indeed the result of imitated arm lifting. Given that our basic assumption is that individuals will imitate the observed movement, this aspect is important. To test whether participants really imitated the movement of the athlete in the arm lifting condition, we changed the procedure for Study 3. In contrast to the previous two studies, the test drink was served in many small drinking cups rather than in one large cup. Thus, participants had to repeat the arm lifting movement to bring the cups to their mouths. Moreover, this method allows for a simple measurement of arm lifting frequency through counting the number of cups used. We argue that participants' drink intake is the result of imitating the movements of the athlete as specified in the following hypothesis:

Hypothesis 3: Participants watching the compatible arm movement (arm lifting) will raise more cups to their mouths and therefore drink more than participants who watch the incompatible arm movement (arm pushing).

\section{Method}

Participants. Thirty-one students (six men and 25 women) completed the study in exchange for partial course credit and were tested in groups of up to five persons. The participants' ages ranged from 19 to 35 years with a mean age of $22.24(S D=2.90)$.

Procedure. The procedure of Study 3 was similar to that applied in Study 2 with the following exceptions: In contrast to the previous studies, the participants watched the videos from the first-person perspective only and did not drink from a large cup, but from many small cups. Ten plastic cups, each with a capacity of $20 \mathrm{ml}$, were placed in front of every participant. With a pipette, the experimenter dripped exactly $7 \mathrm{ml}$ of an ice-tea-flavored drink into each cup. Participants were told that drinking in small sips is a widely known method for amplifying one's taste experience. While tasting the beverage, they were shown the first-person perspective videos used in Study 2 with an athlete either lifting (compatible arm movement) or pushing a barbell (incompatible arm movement). To ensure that participants had enough time to use different cups, the duration of the videos was twice as long (80 s) as in the other studies.

\section{Results}

Drink intake. None of the participants guessed the purpose of the experiment. In line with our hypothesis, participants in the compatible arm movement condition used more cups $(M=5.53$, $S D=1.96)$ and drank more of the beverage $(M=38.73 \mathrm{ml}, S D=$ 13.71) than participants in the incompatible arm movement condition $\left(M_{\text {cups }}=3.06, S D=1.81 ; M_{\text {drink intake }}=21.44 \mathrm{ml}, S D=\right.$ 12.64), $t(29)=3.65$, all $p \mathrm{~s}<.001$, all $d \mathrm{~s}>0.53$.

Drink evaluation. In an additional analysis, we tested whether the observed movements had an effect on drink evaluation. The mean of the three drink evaluation items (Cronbach's 
$\alpha=.95)$ served as the dependent variable. A $t$ test for independent samples revealed no differences between the observed movements on the evaluation of the drink, $t(29)=0.16, p=.87$.

\section{Discussion}

Whereas Studies 1 and 2 showed that watching a model perform a movement that is compatible with the drinking movement caused observers to drink more of a beverage, Study 3 provided further support for the presumed underlying imitation process. Watching an athlete lift a barbell toward his head prompted participants to perform similar movements. Observing arm lifting led participants to lift more drinking cups than observing arm pushing.

Although the results of the previous studies supported our hypotheses that were derived from ideomotor theories (Brass \& Heyes, 2005; Greenwald, 1970; James, 1890; Jeannerod, 1999; Prinz, 1997) and findings on the perception-behavior link (Chartrand \& Bargh, 1999; Chartrand et al., 2005; Dijksterhuis \& Bargh, 2001; Dijksterhuis et al., 2005), there was still a need to address whether the observation of movements might prime an approach or avoidance goal rather than facilitate compatible motor responses more directly. Indeed, previous studies have demonstrated that movements similar to those applied in the present research could prime broad schemas of approach or avoidance and affect behaviors and evaluations (e.g., Cacioppo, Priester, \& Berntson, 1993; Förster, 2003, 2004). The basic idea behind this research is that arm flexion is often applied when approaching positive stimuli (e.g., flexion of the arm to grasp food), whereas arm extension is often applied when avoiding negative stimuli (e.g., pushing unpleasant food away) and that, thus, arm flexion primes approach, whereas arm extension primes avoidance. Cacioppo et al. (1993), for instance, asked participants to press their hand either up against the underside of a table (arm flexion) or down onto the surface of the table (arm extension) and found that arm flexion, in comparison to arm extension, led to more positive evaluations of novel stimuli. Similarly, Förster $(2003,2004)$ found that arm flexion in comparison to arm extension increased participants' evaluation of consumption products (Förster, 2004) as well as drink intake (Förster, 2003).

The observed arm lifting (pushing) movement in our studies involves muscles similar to the performed arm flexion (extension) movement reviewed above (Cacioppo et al., 1993; Förster, 2003, 2004). Therefore, it could reasonably be argued that the observation of frequently used arm lifting movements to pull an object toward oneself might activate an approach goal, and the observation of arm pushing movements to push something away from oneself might activate an avoidance goal in the observer. In our previous studies, these activated goals then might have caused an increase or decrease in drink intake. At this point, we cannot rule out this alternative explanation. However, one finding might speak against an activation of broad approach or avoidance concepts: We did not find effects of the videos on the evaluation of the drinks in any study. If general concepts of approach and avoidance had been primed, one would assume that these activations should have had an effect on the evaluations of the target also. Indeed, effects of arm extension and arm flexion on evaluation were observed in the studies by Cacioppo et al. (1993 and Förster (2004), but not in the present studies. The fact that we did not find an effect of the observed movements on evaluation suggests that the observed movement directly triggered the respective behavior without affecting the evaluation of the drinks. Hence, the present studies are in line with the notion that imitation processes underlie the observed effects. Nevertheless, a more systematic study is necessary to rule out the possibility that processes of priming approach versus avoidance could be responsible for the effects of the observation of arm lifting versus arm pushing on drink consumption. Study 4 was designed to address this concern. An approach versus avoidance priming account would predict an increase in drink intake regardless of the mode of drinking. However, an imitation account would assume that drink intake is affected only by drinking modes in which arm lifting is involved and not by other modes of drinking (e.g., drinking with a straw).

\section{Study 4}

To test whether the observation of an actor executing an arm lifting movement affects consumption behavior by indeed eliciting imitation processes or alternatively by priming approach and avoidance behaviors, we varied the procedure used for drink intake in Study 4. The videos presented were the same videos taken from the first-person perspective as in Studies 2 and 3. But while watching the videos, half the participants drank out of a plastic cup and the other half drank from a tube. Drinking from a tube does not involve any arm movements. If observing a compatible versus incompatible movement affects consumption behavior through imitation, the observation of a movement should affect only behaviors that involve compatible movements and not behaviors that involve unrelated movements. Thus, the observation of arm lifting compared to the observation of arm pushing should affect drinking behavior based on lifting a cup to the mouth, but not drinking from a tube. By contrast, if observing arm lifting or pushing affects drinking behavior by priming approach or avoidance schemata that are transformed into behavior, the manner of consumption should be irrelevant, and arm lifting should lead to more drinking than arm pushing irrespective of whether a cup or a tube is used for drinking. Because we assume that imitation processes underlie the effects of the observation of movements on drink consumption, we formulated the following hypothesis:

Hypothesis 4: Observers drinking out of a cup will drink more when watching arm lifting (compatible arm movement) than when watching arm pushing (incompatible arm movement). However, this effect will not occur when observers drink from a tube.

\section{Method}

Participants and design. Eighty-six students (16 men and 70 women) between the ages of 18 and 48 years $(M=22.13, S D=$ 4.77) participated in exchange for partial course credit. They were tested in groups of up to three persons. The experiment had a 2 (movement: arm lifting vs. arm pushing) $\times 2$ (mode of drink intake: cup vs. tube) between-subjects design. We assigned participants randomly to the two movement conditions and varied the drink intake condition between sessions to ensure that participants did not guess the hypothesis.

Procedure. The procedure used in Study 4 was similar to that applied in the previous studies. Participants watched a video with 
an actor executing either arm lifting with a barbell or arm pushing with a barbell. All videos showed the first-person perspective of the actor. However, in contrast to the other studies, we applied two drink intake conditions. Half the participants drank out of a plastic cup and the other half from a tube (see Figure 2). To implement the tube condition, we made use of hydration systems, which are often used in bicycling or running. A mouthpiece was connected via a tube to a container holding the beverage. The container had the same capacity as the plastic cups $(200 \mathrm{ml})$ used in the cup condition and was mounted beneath the table where the participants were sitting. As a cover story, the experimenter told the participants that a new sports drink would come onto the market. To simulate a sports context in which the novel sports drink would be implemented, we showed them a video of an athlete exercising with a barbell during which they had to drink out of a tube (cup), which is often used in different areas of sports. Before watching the video, participants in the tube condition were instructed to bite on the mouthpiece and to suck until they felt the first droplet in their mouth. They were then told to hold down their arms and to start the video by clicking the next button. In the cup condition, they were told to grasp the cup and to start the video by pressing the next button. All participants were allowed to start drinking as soon as the video began and were instructed to drink as much as they wanted to of the white-tea-flavored beverage. Afterward, participants evaluated the drink on the same items as used in the prior studies and were then probed for suspicion and debriefed.

\section{Results}

Drink intake. None of the participants guessed the purpose of the experiment. According to an imitation account, the movements of the actor presented in the videos would have an impact on drink intake only when participants consumed the drink with a cup, but not when they consumed it by a tube. By contrast, an approachavoidance account would not be able to explain why the effect should be moderated by the means of consumption.

To investigate our hypotheses, we computed a 2 (movement: arm lifting vs. arm pushing) $\times 2$ (mode of drink intake: cup vs. tube) ANOVA with the amount consumed as the dependent variable. As expected, the ANOVA revealed a significant interaction between movement and mode of drink intake, $F(1,82)=5.22$, $p=.03, \eta_{\mathrm{p}}^{2}=.06$. The main effect of mode of drink intake, $F(1$,
$82)=0.31, p=.58$, was not significant, and the main effect of observed body movement, $F(1,82)=3.11 p=.08$, was marginally significant. Separate analyses revealed that participants in the cup condition drank more of the offered drink when they had watched the arm lifting video $(M=168.71 \mathrm{~g}, S D=97.72)$ than when they had watched the arm pushing video $(M=96.14 \mathrm{~g}$, $S D=63.90), t(70)=2.86, p=.005, d=0.87$. By contrast, when participants drank from the tube, there was no difference between the observed body movement conditions $\left(M_{\text {lifting }}=117.72 \mathrm{~g}\right.$, $\left.S D=84.79 ; M_{\text {pushing }}=127.05 \mathrm{~g}, S D=83.23\right), t(70)=-0.37$, $p=.71$.

Drink evaluation. In a further analysis, we investigated whether the observed movement and the mode of drinking affected the evaluation of the drink. First, we computed a mean composite score of all the drink-evaluation items (Cronbach's $\alpha=.93$ ). A 2 (movement: arm lifting vs. arm pushing) $\times 2$ (mode of drink intake: cup vs. tube) ANOVA with the mean composite of the drink evaluation as the dependent variable revealed no significant interaction or main effects, $F_{\mathrm{s}}(1,82)<2.23, p \mathrm{~s}>.14$.

\section{Discussion}

Study 4 aimed to test the assumed imitation account underlying our effects in more detail by varying the mode of drinking. The results support our assumption because the observed arm lifting movement had an effect on drink intake only when drinking itself relied on arm lifting. When drinking was realized by other means, the observed movements had no effect on drink intake. It is important to note that these results are inconsistent with an approach versus avoidance priming explanation. If the arm lifting movement had primed an approach, and the arm pushing movement an avoidance concept, the observation of the arm movements would have had an effect on the drink consumption in any mode of drink intake.

The findings of Study 4 also rule out an alternative explanation that we have not discussed so far. One might speculate that the arm lifting movement is perceived as being more exhausting and therefore causing more thirst. However, because we found no differences in drink intake between the two movements in the tube condition, this explanation is unlikely to account for the observed effects.

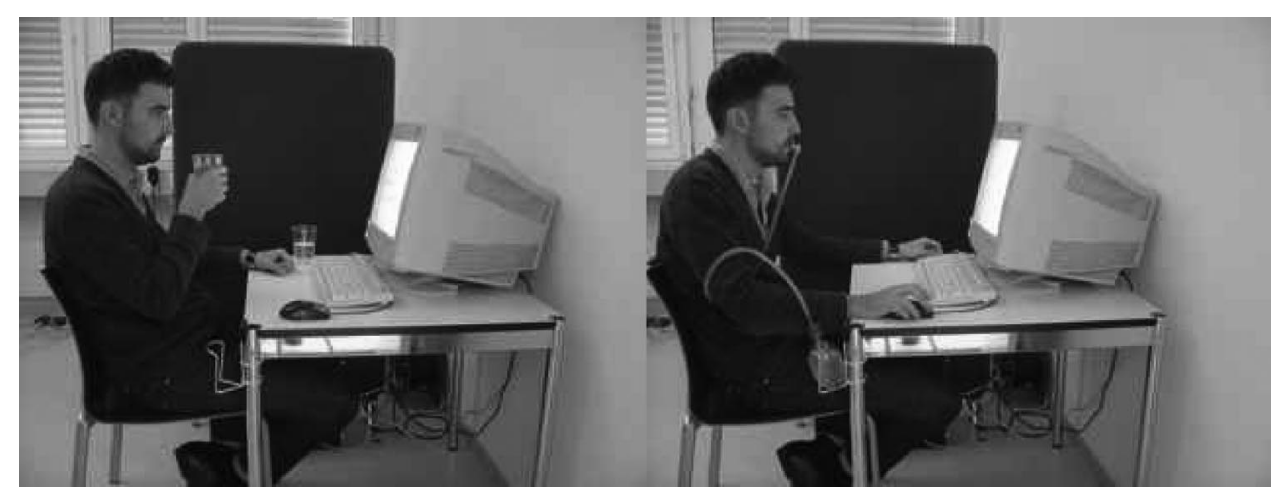

Figure 2. Schematic depiction of the two drink intake conditions (plastic cup vs. tube) from Study 4. 


\section{General Discussion}

We assumed that watching a movement in one behavioral context can stimulate the imitation of the movement in a different behavioral context. We tested this assumption and explored a possible consumer consequence of this cross-contextual imitation in the context of drink intake. In four studies with different drinks, we showed that watching a video of an athlete lifting a barbell compared to pushing a barbell led participants to raise a cup toward their mouths more often and thus to increase their drink intake. Furthermore, and in line with previous studies on imitation (e.g., Chartrand \& Bargh, 1999; Jackson et al., 2006; Lamm et al., 2007; Lamm et al., 2008), we found that the perspective of the participants moderated this movement compatibility effect.

These results fit nicely with theories proposing an overlap between movement observation and movement execution (i.e., the perception-behavior link and ideomotor theories). For example, in the Brass et al. (2001, 2000) studies, participants were instructed to perform a certain movement when a picture was displayed on a screen. A consistent finding was that participants responded more quickly to such pictures that displayed a movement compatible with the preinstructed movement. Theories proposing an overlap between movement observation and movement execution explain this effect with the notion that the observation of a certain movement activates the compatible movement and therefore facilitates its execution. By contrast, when an incompatible movement is observed, it is assumed that the incompatible response is activated, which thus makes inhibition more likely. We assume that similar processes are involved in our experiments. Specifically, we suppose that watching an athlete perform a movement compatible with a drinking movement facilitates the execution of this movement, which results in more frequent cup lifting and consequently leads to an increase in drink intake. This is in line with neurophysiological research that has demonstrated that observing a specific movement activates muscles similar to the ones used when executing this movement (Berger \& Hadley, 1975; Fadiga et al., 1995). Therefore, it is reasonable to assume that in our experiments, the subtle activation of muscles facilitated the execution of the drinking behavior when compatible movements were observed.

We also found stronger effects of the observation of compatible movements when participants took the perspective of the observed actor because of an inclination to do so or because participants were made to take the perspective of the observer by the way the video was filmed. In Study 1, participants imitated the movement more strongly the more they took the perspective of the athlete. In Study 2, participants watching the first-person perspective video imitated the athlete more strongly than participants watching the third-person video. These findings are very much in line with recent research showing that an individual's perspective is an important moderator of imitation (e.g., Chartrand \& Bargh, 1999; Jackson et al., 2006; Lamm et al., 2007, 2008). Jackson et al. (2006), for example, showed participants videos taken from a first-person and from a third-person perspective. Observing a movement from the first-person perspective resulted in shorter latencies for imitating the movement than observing the movement from the external perspective. Moreover, functional imaging results demonstrated that the first-person perspective was more tightly coupled to the sensorimotor system than the third-person perspective.

\section{Theoretical Implications and Future Directions}

The idea that individuals mirror the behavior of others is not new. The novel contribution of our research is that imitation is not specific to the context in which the behavior is observed, but even occurs across behavioral contexts and across different goals. To our knowledge, existing research on imitation and mimicry has shown effects only for behavior that was performed with the same goal and in the same context as the observed action.

Whereas our finding of cross-contextual imitation supports theories proposing that the observation of certain movements activates motor programs similar to when executing the movement (for reviews, see Chartrand et al., 2005; Meltzoff \& Prinz, 2002), it also extends and complements theories on goal-directed imitation (Bekkering et al., 2000; Gattis et al., 2002; Gleissner et al., 2000). According to the latter theories, individuals extract the goal of the observed movement when observing others and imitate the goal with an action that easily allows the pursuit of the goal, irrespective of whether this action requires a movement similar to the observed movement. There is no doubt that goal representation is an important factor for imitation, but our findings suggest-in line with other research on imitation (Bird, Brindley, Leighton, \& Heyes, 2007; Leighton, Bird, \& Heyes, 2010)—that goal representation is not a necessary precondition for imitation per se. Whereas some studies suggest that goal representation is not a necessary precondition for imitation in situations in which attention is directed toward motor control (Leighton et al., 2010), our results demonstrate that in situations of high ideomotor compatibility, goal representation is not necessary for imitating a certain movement. This notion also has important implications for more general models of behavior (Bargh \& Ferguson, 2000; Powers, 1973). Whereas these models highlight the importance of goals in behavior, our research emphasizes the underlying motor actions that are needed to reach the goal. For an individual, the goal of reaching a certain end state (e.g., making a basket in basketball) itself will not lead to a successful end state without having control over its underlying motor actions (e.g., shooting the ball properly).

Besides the implications for imitation research and more general models of behaviors, our results furthermore have important implications for research on the effects of environmental cues on food and drink intake. Recent research has shown that individuals often adjust their amount of consumption based on environmental cues (for a review, see van't Riet, Sijtsema, Dagevos, \& De Bruijn, 2011). For example, people adjust their food intake according to the size of food packaging (Rolls, Roe, Kral, Meengs, \& Wall, 2004) or the size of food portions (Wansink, Painter, \& North, 2005). Further, and more relevant to our research, an important environmental influence on food and drink intake is the behavior of others (for an overview, see Herman et al., 2003). The consumption behavior of individuals consuming together is often highly correlated. If one person eats or drinks a large amount, the other person will also tend to eat and drink more. By contrast, if one person consumes less, the other person is more likely to also consume less. One of the most prominent explanations for this effect is socially derived norms (e.g., Herman et al., 2003). Our results suggest, however, that at least to some degree, adjusted food intake is also affected by imitating a specific movement.

Because we assume that increased drink intake is caused by a direct link between the observation of a movement and the exe- 
cution of the same movement, it would be consistent to assume that individuals are not aware of the effect. Indeed, in our studies, no participant correctly guessed our hypotheses or mentioned that she or he imitated the actor. An interesting question, though, is whether individuals can resist this kind of unconscious influence if becoming aware of the source of the influence-in our case, the link between the perception and the execution of an arm lifting movement. Several studies have shown that effects on judgments and evaluations are reduced when individuals become aware of the source of influence (e.g., Schwarz \& Clore, 1983). For example, Schwarz and Clore (1983) found that individuals relied on their current mood when they judged their life satisfaction. However, these effects disappeared when their attention was drawn to the source of their mood. In our case, because the imitated behavior may not be regarded as diagnostic information, individuals could correct the influence of this behavior if their attention was directed to it. Future research may explore whether a forewarning of imitation could weaken its influence on consumption.

\section{Conclusion}

Imitation is a well-known phenomenon, and its effects have been typically shown when the imitated and the imitating behavior pursued the same goal (for reviews, see Chartrand et al., 2005; Meltzoff \& Prinz, 2002). Adding to this literature, our research suggests that imitation may occur more frequently and may represent a broader phenomenon than previously assumed if imitating is not confined to the same class of behaviors but rather to the same class of movements that may be involved in different behaviors. This finding therefore emphasizes the pervasiveness of imitation and demonstrates the power of the movement in imitation processes.

\section{References}

Aiken, L. S., \& West, S. G. (1996). Multiple regression testing and interpreting interactions. London, England: Sage.

Bargh, J. A., \& Ferguson, M. J. (2000). Beyond behaviorism: On the automaticity of higher mental processes. Psychological Bulletin, 126, 925-945. doi:10.1037/0033-2909.126.6.925

Barsalou, L. W. (1999). Perceptual symbol systems. Behavioral and Brain Sciences, 22, 577-660.

Bekkering, H., Wohlschläger, A., \& Gattis, M. (2000). Imitation of gestures in children is goal-directed. Quarterly Journal of Experimental Psychology: Human Experimental Psychology, 53A, 153-164. doi: 10.1080/713755872

Berger, S. M., \& Hadley, S. W. (1975). Some effects of a model's performance on an observer's electromyographic activity. American Journal of Psychology, 88, 263-276. doi:10.2307/1421596

Bird, G., Brindley, R., Leighton, J., \& Heyes, C. (2007). General processes, rather than "goals," explain imitation errors. Journal of Experimental Psychology: Human Perception and Performance, 33, 1158-1169. doi: 10.1037/0096-1523.33.5.1158

Brass, M., Bekkering, H., \& Prinz, W. (2001). Movement observation affects movement execution in a simple response task. Acta Psychologica, 106, 3-22. doi:10.1016/S0001-6918(00)00024-X

Brass, M., Bekkering, H., Wohlschläger, A., \& Prinz, W. (2000). Compatibility between observed and executed finger movements: Comparing symbolic, spatial, and imitative cues. Brain and Cognition, 44, 124-143. doi:10.1006/brcg.2000.1225
Brass, M., \& Heyes, C. (2005). Imitation: Is cognitive neuroscience solving the correspondence problem? Trends in Cognitive Sciences, 9, 489-495. doi:10.1016/j.tics.2005.08.007

Byrne, R. W. (2003). Imitation as behaviour parsing. Philosophical Transactions of the Royal Society of London Series B: Biological Sciences, 358, 529-536. doi:10.1098/rstb.2002.1219

Byrne, R. W., \& Byrne, J. M. (1991). Hand preferences in the skilled gathering tasks of mountain gorillas (Gorilla g. beringei). Cortex, 27, 521-546.

Cacioppo, J. T., Priester, J. R., \& Berntson, G. G. (1993). Rudimentary determinants of attitudes: II. Arm flexion and extension have differential effects on attitudes. Journal of Personality and Social Psychology, 65, 5-17. doi:10.1037/0022-3514.65.1.5

Carpenter, M., Akhtar, N., \& Tomasello, M. (1998). Fourteen- through 18-month-old infants differentially imitate intentional and accidental actions. Infant Behavior \& Development, 21, 315-330. doi:10.1016/ S0163-6383(98)90009-1

Chartrand, T. L., \& Bargh, J. A. (1999). The chameleon effect: The perception-behavior link and social interaction. Journal of Personality and Social Psychology, 76, 893-910. doi:10.1037/0022-3514.76.6.893

Chartrand, T. L., Maddux, W. W., \& Lakin, J. L. (2005). Beyond the perception-behavior link: The ubiquitous utility and motivational moderators of nonconscious mimicry. In R. R. Hassin, J. S. Uleman, \& J. A. Bargh (Eds.), The new unconscious (pp. 334-361). New York, NY: Oxford University Press. doi:10.1093/acprof:oso/9780195307696.003 .0014

Conger, J. C., Conger, A. J., Costanzo, P. R., Wright, K. L., \& Matter, J. A. (1980). The effect of social cues on the eating behavior of obese and normal subjects. Journal of Personality, 48, 258-271. doi:10.1111/j $.1467-6494.1980 . t b 00832 . x$

Dijksterhuis, A., \& Bargh, J. A. (2001). The perception-behavior expressway: Automatic effects of social perception on social behavior. Advances in Experimental Social Psychology, 33, 1-40. doi:10.1016/ S0065-2601(01)80003-4

Dijksterhuis, A., Smith, P. K., van Baaren, R. B., \& Wigboldus, D. H. J. (2005). The unconscious consumer: Effects of environment on consumer behavior. Journal of Consumer Psychology, 15, 193-202. doi:10.1207/ s15327663jcp1503_3

Fadiga, L., Fogassi, L., Pavesi, G., \& Rizzolatti, G. (1995). Motor facilitation during action observation: A magnetic stimulation study. Journal of Neurophysiology, 73, 2608-2611.

Förster, J. (2003). The influence of approach and avoidance motor actions on food intake. European Journal of Social Psychology, 33, 339-350. doi:10.1002/ejsp. 150

Förster, J. (2004). How body feedback influences consumers' evaluation of products. Journal of Consumer Psychology, 14, 416-426. doi:10.1207/ s15327663jcp1404_10

Gattis, M., Bekkering, H., \& Wohlschläger, A. (2002). Goal-directed imitation. In A. N. Meltzoff \& W. Prinz (Eds.), The imitative mind: Development, evolution, and brain bases (pp. 183-205). New York, NY: Cambridge University Press. doi:10.1017/CBO9780511489969.011

Gergely, G., Bekkering, H., \& Kiraly, I. (2002). Developmental psychology: Rational imitation in preverbal infants. Nature, 415, 755. doi: $10.1038 / 415755 \mathrm{a}$

Gleissner, B., Bekkering, H., \& Meltzoff, A. N. (2000). Children`s coding of human action: Cognitive factors influencing imitation in 3-year-olds. Developmental Science, 3, 405-414. doi:10.1111/1467-7687.00135

Goldman, S. J., Herman, C. P., \& Polivy, J. (1991). Is the effect of a social model on eating attenuated by hunger? Appetite, 17, 129-140. doi: 10.1016/0195-6663(91)90068-4

Greenwald, A. G. (1970). Sensory feedback mechanisms in performance control: With special reference to the ideo-motor mechanism. Psychological Review, 77, 73-99. doi:10.1037/h0028689 
Grossman, E., Donnelly, M., Price, R., Pickens, D., Morgan, V., Neighbor, G., \& Blake, R. (2000). Brain areas involved in perception of biological motion. Journal of Cognitive Neuroscience, 12, 711-720. doi:10.1162/ 089892900562417

Herman, C. P., Koenig-Nobert, S., Peterson, J. B., \& Polivy, J. (2005). Matching effects on eating: Do individual differences make a difference? Appetite, 45, 108-109. doi:10.1016/j.appet.2005.03.013

Herman, C. P., Roth, D. A., \& Polivy, J. (2003). Effects of the presence of others on food intake: A normative interpretation. Psychological Bulletin, 129, 873-886. doi:10.1037/0033-2909.129.6.873

Heyes, C. (2001). Causes and consequences of imitation. Trends in Cognitive Sciences, 5, 253-261. doi:10.1016/S1364-6613(00)01661-2

Heyes, C. (2011). Automatic imitation. Psychological Bulletin, 137, 463483. doi:10.1037/a0022288

Huang, C. T., \& Charman, T. (2005). Gradations of emulation learning in infants' imitation of actions on objects. Journal of Experimental Child Psychology, 92, 276-302. doi:10.1016/j.jecp.2005.06.003

Jackson, P. L., Meltzoff, A. N., \& Decety, J. (2006). Neural circuits involved in imitation and perspective-taking. NeuroImage, 31, 429-439. doi:10.1016/j.neuroimage.2005.11.026

James, W. (1890). The principles of psychology (Vol. 2). New York, NY: Holt. doi:10.1037/11059-000

Jeannerod, M. (1999). The 25th Bartlett Lecture: To act or not to act: Perspectives on the representation of actions. Quarterly Journal of Experimental Psychology: Human Experimental Psychology, 52A, 1-29. doi:10.1080/027249899391205

Johnston, L. (2002). Behavioral mimicry and stigmatization. Social Cognition, 20, 18-35. doi:10.1521/soco.20.1.18.20944

Lamm, C., Batson, C. D., \& Decety, J. (2007). The neural substrate of human empathy: Effects of perspective-taking and cognitive appraisal. Journal of Cognitive Neuroscience, 19, 42-58. doi:10.1162/jocn.2007 .19.1.42

Lamm, C., Porges, E. C., Cacioppo, J. T., \& Decety, J. (2008). Perspective taking is associated with specific facial responses during empathy for pain. Brain Research, 1227, 153-161. doi:10.1016/j.brainres.2008.06 .066

Leighton, J., Bird, G., \& Heyes, C. (2010). "Goals" are not an integral component of imitation. Cognition, 114, 423-435. doi:10.1016/j .cognition.2009.11.001

Leighton, J., \& Heyes, C. (2010). Hand to mouth: Automatic imitation across effector systems. Journal of Experimental Psychology: Human Perception and Performance, 36, 1174-1183. doi:10.1037/a0019953

Lingnau, A., Gesierich, B., \& Caramazza, A. (2009). Asymmetric fMRI adaptation reveals no evidence for mirror neurons in humans. Proceedings of the National Academy of Sciences of the United States of America, 106, 9925-9930. doi:10.1073/pnas.0902262106

Meltzoff, A. N. (1995). Understanding the intentions of others: Reenactment of intended acts by 18-month-old children. Developmental Psychology, 31, 838-850. doi:10.1037/0012-1649.31.5.838
Meltzoff, A. N., \& Prinz, W. (Eds.). (2002). The imitative mind: Development, evolution, and brain bases. New York, NY: Cambridge University Press. doi:10.1017/CBO9780511489969

Montastruc, P. (1986). L'alcool exagere la soif [Alcohol exaggerates thirst]. HCEIA Informations, 4, 41-42.

Nicholson, W. M., \& Taylor, H. M. (1938). The effect of alcohol on the water and electrolyte balance in man. Journal of Clinical Investigation, 17, 279-285. doi:10.1172/JCI100951

Niedenthal, P. M., Barsalou, L. W., Winkielman, P., Krauth-Gruber, S., \& Ric, F. (2005). Embodiment in attitudes, social perception, and emotion. Personality and Social Psychology Review, 9, 184-211. doi:10.1207/ s15327957pspr0903_1

Nisbett, R. E., \& Storms, M. D. (1974). Cognitive and social determinants of food intake. In R. E. Nisbett (Ed.), Thought and feeling: Cognitive alteration of feeling states (pp. 190-208). Chicago, IL: Aldine.

Powers, W. T. (1973). Feedback: Beyond behaviorism. Science, 179, 351-356. doi:10.1126/science.179.4071.351

Prinz, W. (1990). A common coding approach to perception and action. In O. Neumann \& W. Prinz (Eds.), Relationships between perception and action (pp. 167-201). Berlin, Germany: Springer-Verlag. doi:10.1007/ 978-3-642-75348-0 7

Prinz, W. (1997). Perception and action planning. European Journal of Cognitive Psychology, 9, 129-154. doi:10.1080/713752551

Rizzolatti, G., \& Sinigaglia, C. (2010). The functional role of the parietofrontal mirror circuit: Interpretations and misinterpretations. Nature Reviews Neuroscience, 11, 264-274. doi:10.1038/nrn2805

Rolls, B. J., Roe, L. S., Kral, T. V. E., Meengs, J. S., \& Wall, D. E. (2004), Increasing the portion size of a packaged snack increases energy intake in men and women. Appetite, 42, 63-69. doi:10.1016/S01956663(03)00117-X

Ruby, P., \& Decety, J. (2001). Effect of subjective perspective taking during simulation of action: A PET investigation of agency. Nature Neuroscience, 4, 546-550.

Schwarz, N., \& Clore, G. L. (1983). Mood, misattribution, and judgments of well-being: Informative and directive functions of affective states. Journal of Personality and Social Psychology, 45, 513-523. doi: 10.1037/0022-3514.45.3.513

Storms, M. D. (1973). Videotape and attribution process: Reversing actors and observers' points of view. Journal of Personality and Social Psychology, 27, 165-175. doi:10.1037/h0034782

van't Riet, J., Sijtsema, S. J., Dagevos, H., \& De Bruijn, G.-J. (2011). The importance of habits in eating behaviour. An overview and recommendations for future research. Appetite, 57, 585-596. doi:10.1016/j.appet 2011.07.010

Wansink, B., Painter, J. E., \& North, J. (2005). Bottomless bowls: Why visual cues of portion size may influence intake. Obesity Research, 13, 93-100. doi:10.1038/oby.2005.12

Received December 6, 2011

Revision received June 17, 2012

Accepted July 19, 2012 